PROCEEDINGS OF THE

AMERICAN MATHEMATICAL SOCIETY

Volume 127, Number 6, Pages 1819-1826

S 0002-9939(99)05014-5

Article electronically published on February 18, 1999

\title{
THE CREMMER-GERVAIS SOLUTION OF THE YANG-BAXTER EQUATION
}

\author{
TIMOTHY J. HODGES
}

(Communicated by Ken Goodearl)

\begin{abstract}
A direct proof is given of the fact that the Cremmer-Gervais $R$ matrix satisfies the (Quantum) Yang-Baxter equation
\end{abstract}

\section{INTRODUCTION}

Let $V$ be a vector space of rank $n$ over a field $F$. Let $c \in$ End $V \otimes V$ be a linear operator. Define $c_{12}, c_{23} \in$ End $V \otimes V \otimes V$ by $c_{12}=c \otimes I d, c_{23}=I d \otimes c$. Then $c$ is said to satisfy the Yang-Baxter equation (YBE) if

$$
c_{12} c_{23} c_{12}=c_{23} c_{12} c_{23} \text {. }
$$

An extremely interesting solution of this equation was found by Cremmer and Gervais in their paper [1]. In its slightly more general two parameter form, it is (up to a scalar)

$$
c\left(e_{i} \otimes e_{j}\right)= \begin{cases}q e_{j} \otimes e_{i} & \text { if } i=j, \\ q p^{i-j} e_{j} \otimes e_{i}+\sum_{i \leq k<j}\left(q-q^{-1}\right) p^{i-k} e_{k} \otimes e_{i+j-k} & \text { if } i<j, \\ q^{-1} p^{i-j} e_{j} \otimes e_{i}+\sum_{j<k<i}\left(q^{-1}-q\right) p^{i-k} e_{k} \otimes e_{i+j-k} & \text { if } i>j,\end{cases}
$$

where $\left\{e_{1}, \ldots, e_{n}\right\}$ is a basis for $V$, and $q$ and $p$ are non-zero elements of $F$. Taking $q=p^{n / 2}$ yields the original operator given by Cremmer and Gervais. The derivation of this solution used some fairly technical calculations involving chiral vertex operators and is a little inaccessible to the non-specialist. Here we give an elementary proof of this result along the same lines as the proof in [3] for the standard solutions of the Yang-Baxter equation.

\section{Linear Combinations of SOlutions of the YBE}

Suppose $f$ and $g$ are solutions of the YBE and let $\alpha, \beta \in F$. Expanding the equation

$$
(\alpha f+\beta g)_{12}(\alpha f+\beta g)_{23}(\alpha f+\beta g)_{12}=(\alpha f+\beta g)_{23}(\alpha f+\beta g)_{12}(\alpha f+\beta g)_{23},
$$

Received by the editors September 19, 1997.

1991 Mathematics Subject Classification. Primary 81R50, 17B37; Secondary 16W30.

Key words and phrases. Quantum group, $R$-matrix.

The author was supported in part by a grant from the National Science Foundation.

(C)1999 American Mathematical Society 
we see that $c=\alpha f+\beta g$ will be a solution of the YBE for all $\alpha, \beta \in A$ if the following two conditions are satisfied:

$$
\begin{aligned}
& f_{12} g_{23} g_{12}+g_{12} f_{23} g_{12}+g_{12} g_{23} f_{12}=f_{23} g_{12} g_{23}+g_{23} f_{12} g_{23}+g_{23} g_{12} f_{23}, \\
& g_{12} f_{23} f_{12}+f_{12} g_{23} f_{12}+f_{12} f_{23} g_{12}=g_{23} f_{12} f_{23}+f_{23} g_{12} f_{23}+f_{23} f_{12} g_{23} .
\end{aligned}
$$

In the case where $f$ is the permutation operator $P\left(e_{i} \otimes e_{j}\right)=e_{j} \otimes e_{i}$, the second condition is true for any $g$ (since $g_{12} P_{23} P_{12}=P_{23} P_{12} g_{23}$ and similar equalities hold for the other terms). Thus we obtain the following simple condition which we shall refer to as the compatibility condition.

Lemma 2.1. Suppose that $g \in$ End $V \otimes V$ is a solution of the $Y B E$. Then $c=$ $\alpha P+\beta g$ will be a solution of the YBE for all $\alpha, \beta \in F$ if

$$
g_{12} g_{23} P_{12}+g_{12} P_{23} g_{12}+P_{12} g_{23} g_{12}=g_{23} g_{12} P_{23}+g_{23} P_{12} g_{23}+P_{23} g_{12} g_{23} \text {. }
$$

We shall apply this result to the case where

$$
g\left(e_{i} \otimes e_{j}\right)=\sum_{k} \eta(i, j, k) e_{k} \otimes e_{i+j-k}
$$

and

$$
\eta(i, j, k)= \begin{cases}1 & \text { if } i \leq k<j \\ -1 & \text { if } j \leq k<i \\ 0 & \text { otherwise }\end{cases}
$$

Taking $\alpha=q$ and $\beta=\left(q-q^{-1}\right)$ yields

$$
c\left(e_{i} \otimes e_{j}\right)= \begin{cases}q e_{j} \otimes e_{i} & \text { if } i=j, \\ q e_{j} \otimes e_{i}+\sum_{i \leq k<j}\left(q-q^{-1}\right) e_{k} \otimes e_{i+j-k} & \text { if } i<j, \\ q^{-1} e_{j} \otimes e_{i}+\sum_{j<k<i}\left(q^{-1}-q\right) e_{k} \otimes e_{i+j-k} & \text { if } i>j,\end{cases}
$$

which is the Cremmer-Gervais operator described in the introduction in the case where $p=1$. Once we have shown that this operator satisfies the Yang-Baxter equation, it follows from some well-known "twisting" results [2] that the more general operator is also a solution.

\section{The COMPATIBILITY CONDITION}

In this section we check the compatibility condition (2.1) for the operator $g$ given above.

Lemma 3.1. Let $g \in$ End $V \otimes V$ be an operator of the form

$$
g\left(e_{i} \otimes e_{j}\right)=\sum_{k} \eta(i, j, k) e_{k} \otimes e_{i+j-k},
$$

where $\eta(i, j, k)=0$ if $k$ is not between $i$ and $j$. Then the condition of Lemma 2.1 is satisfied if and only if

$$
\begin{aligned}
& \eta(i, k, a+b-j) \eta(j, a+b-j, a)+\eta(i, j, b+a-k) \eta(b+a-k, k, a) \\
& \quad+\eta(i, j, b) \eta(i+j-b, k, a)=\eta(i, k, a) \eta(i+k-a, j, b) \\
& \quad+\eta(j, k, a) \eta(i, j+k-a, b)+\eta(j, k, j+k-b) \eta(i, j+k-b, a)
\end{aligned}
$$

for all $i, j, k, a, b \in\{1,2, \ldots, n\}$. 
Proof. Let $d_{l}=g_{12} g_{23} P_{12}+g_{12} P_{23} g_{12}+P_{12} g_{23} g_{12}$ and $d_{r}=g_{23} g_{12} P_{23}+g_{23} P_{12} g_{23}+$ $P_{23} g_{12} g_{23}$. Denote $e_{i} \otimes e_{j} \otimes e_{k}$ by $[i j k]$. Then

$$
\begin{aligned}
d_{l}[i j k] & =\sum_{s, t} \eta(i, k, t) \eta(j, t, s)[s, j+t-s, i+k-t] \\
& +\sum_{s, t} \eta(i, j, s) \eta(s, k, t)[t, s+k-t, i+j-s] \\
& +\sum_{s, t} \eta(i, j, s) \eta(i+j-s, k, t)[t, s, i+j+k-s-t]
\end{aligned}
$$

and

$$
\begin{aligned}
d_{r}[i j k] & =\sum_{s, t} \eta(i, k, s) \eta(k+i-s, j, t)[s, t, i+j+k-s-t] \\
& +\sum_{s, t} \eta(j, k, s) \eta(i, j+k-s, t)[s, t, i+j+k-t-s] \\
& +\sum_{s, t} \eta(j, k, s) \eta(i, t, s)[s, j+k-t, i+t-s] .
\end{aligned}
$$

Comparing the coefficients of $[a, b, i+j+k-a-b]$ then yields the result.

Now set

$$
u(x)= \begin{cases}1 & \text { if } x \geq 0 \\ 0 & \text { if } x<0\end{cases}
$$

and

$$
\delta(x)= \begin{cases}1 & \text { if } x=0 \\ 0 & \text { if } x \neq 0\end{cases}
$$

Notice that $\eta(i, j, k)=u(k-i)-u(k-j)$.

Lemma 3.2. For any integers $a, b, i, j, k$,

$$
\begin{aligned}
& u(a+b-i-j)(u(a-j)+u(b-i)-u(b-j)-u(j-b))+u(k-b) u(a+b-i-k)) \\
= & u(a-i)(u(k-b)-u(j-b)-u(b-j)+u(b+a-i-k))+u(b-i) u(a-j) .
\end{aligned}
$$

Proof. First note that

$$
u(x)+u(-x)=1+\delta(x)
$$

and

$$
u(x+y)(u(x)+u(y))=u(x) u(y)+u(x+y) .
$$

From this it follows that

$$
\begin{gathered}
u(a+b-i-j)((u(a-j)+u(b-i))=u(a-j) u(b-i)+u(a+b-i-j), \\
u(a+b-i-j)(-u(b-j)-u(j-b))=-u(a+b-i-j)-\delta(b-j) u(a-i)
\end{gathered}
$$


and

$$
\begin{aligned}
u(k-b) u(a+b-i-k)= & (1-u(b-k)+\delta(b-k)) u(a+b-i-k) \\
= & u(a+b-i-k)(1-u(b-k))+\delta(b-k) u(a-i) \\
= & u(a+b-i-k) u(a-i)-u(a-i) u(b-k) \\
& +\delta(b-k) u(a-i) \\
= & u(a+b-i-k) u(a-i)+u(a-i)(u(k-b)-1) .
\end{aligned}
$$

Combining these equations yields the desired result.

Theorem 3.3. For $\eta$ as defined in (2.3), the operator $g\left(e_{i} \otimes e_{j}\right)=\sum_{k} \eta(i, j, k) e_{k} \otimes$ $e_{i+j-k}$ satisifies the compatibility condition (2.1).

Proof. Expanding the left-hand side of (3.1) using $\eta(i, j, k)=u(k-i)-u(k-j)$ yields

$$
\begin{aligned}
& u(a+b-j-k)[u(a-k)-u(k-b)+u(j-b)-u(a-j)] \\
+ & u(a+b-i-j)[-u(j-b)+u(a-j)+u(b-i)-u(b-j)] \\
+ & u(a+b-i-k)[u(k-b)-u(a-k)] \\
+ & u(a-k)[u(b-j)-u(b-i)] .
\end{aligned}
$$

Similarly, the right-hand side becomes

$$
\begin{aligned}
& u(a+b-j-k)[u(a-k)-u(k-b)+u(j-b)-u(a-j)] \\
+ & u(a-k)[u(b-j)-u(b-i)-u(a+b-i-k)] \\
+ & u(a-i)[u(k-b)-u(b-j)-u(j-b)+u(a+b-i-k)] \\
+ & u(b-i) u(a-j) .
\end{aligned}
$$

The equality of these two expressions follows from the identity in Lemma 3.2.

\section{The YANG-BAXTer EQUATION}

In this section we verify that the operator $g\left(e_{i} \otimes e_{j}\right)=\sum_{k} \eta(i, j, k) e_{k} \otimes e_{i+j-k}$ given above satisfies the Yang-Baxter equation. We begin by converting the problem into an identity for $\eta$.

Lemma 4.1. Let $g \in$ End $V \otimes V$ be an operator of the form

$$
g\left(e_{i} \otimes e_{j}\right)=\sum_{k} \eta(i, j, k) e_{k} \otimes e_{i+j-k},
$$

where $\eta(i, j, k)=0$ if $k$ is not between $i$ and $j$. Then $g$ satisfies the Yang-Baxter equation if and only if

$$
\begin{aligned}
\sum_{a} \eta(j, k, a) \eta(i, a, c) & \eta(i+a-c, j+k-a, h) \\
= & \sum_{s} \eta(i, j, s) \eta(i+j-s, k, h+c-s) \eta(s, h+c-s, c)
\end{aligned}
$$

for all $i, j, k, c, h \in\{1,2, \ldots, n\}$.

Proof. The left-hand side of (4.1) is the coefficient of $e_{c} \otimes e_{h} \otimes e_{i+j+k-c-h}$ in the expansion of $g_{23} g_{12} g_{23}\left(e_{i} \otimes e_{j} \otimes e_{k}\right)$. Similarly, the right-hand side is the coefficient of $e_{c} \otimes e_{h} \otimes e_{i+j+k-c-h}$ in $g_{12} g_{23} g_{12}\left(e_{i} \otimes e_{j} \otimes e_{k}\right)$. 
The following identities are used in the proof of the next three results.

Lemma 4.2. For integers $a, b, c, d, e$,

1. $\eta(a+d, b+d, c+d)=\eta(a, b, c)$

2. $\eta(a, b, c)=-\eta(b, a, c)$

3. $\eta(a, b, c)=\eta(-b,-a,-c-1)=\eta(a, b, a+b-c-1)$

4. $\eta(a, a+1, c)=\delta(a-c)$

5. $\sum_{a} \eta(b, c, a)=c-b$

6. $\eta(a, b, d)+\eta(b, c, d)=\eta(a, c, d)$

7. $\eta(a, b+1, c) \eta(c, a, b)=0$

8. $\eta(a, b, c) \eta(c, b, d)=\eta(a, b, d) \eta(a, d+1, c)$

9. $\eta(a, b, c) \eta(d, c, e)=\eta(a, b, c) \eta(d, a, e)+\eta(a, b, e) \eta(e+1, b, c)$.

Proof. The proofs are either trivial or routine calculations.

Lemma 4.3. For any integers, $t, s, b, d, h$,

$$
\begin{aligned}
& \sum_{a} \eta(t, s, a) \eta(b+a, d-a, h)=(s-t) \eta(b+t, d-t, h) \\
& \quad+(d-h-s) \eta(d-s, d-t, h)+(h-b-s+1) \eta(b+t, b+s, h) .
\end{aligned}
$$

Proof. Using the identities of Lemma 4.2,

$$
\begin{aligned}
& \sum_{a} \eta(t, s, a) \eta(b+a, d-a, h) \\
& =-\sum_{a} \eta(t, s, a) \eta(d-b-a, a, h-b) \\
& =-\sum(\eta(t, s, a) \eta(d-b-a, t, h-b)+\eta(t, s, h-b) \eta(h-b+1, s, a)) \\
& =(h-b-s+1) \eta(b+t, b+s, h)-\sum_{a} \eta(t, s, a) \eta(d-b-t, a, d-h-1) .
\end{aligned}
$$

Now

$$
\begin{aligned}
& -\sum_{a} \eta(t, s, a) \eta(d-b-t, a, d-h-1) \\
& =-\sum_{a} \eta(t, s, a) \eta(d-b-t, t, d-h-1)+\eta(t, s, d-h-1) \eta(d-h, s, a) \\
& =(s-t) \eta(t+b-d,-t, h-d)+(d-s-h) \eta(-s,-t, h-d) \\
& =(s-t) \eta(b+t, d-t, h)+(d-h-s) \eta(d-s, d-t, h) .
\end{aligned}
$$

Combining these two equations yields the assertion.

Lemma 4.4. For any integers $i, j, k, c, h$,

$$
\begin{aligned}
& \sum_{a} \eta(j, k, a) \eta(i, a, c) \eta(i+a-c, j+k-a, h) \\
& =\eta(j, k, c)((k-c-1) \eta(i-c+k, j+k-c, h)+(j-h) \eta(j, j+k-c, h) \\
& \quad+(h-i) \eta(i, i+k-c, h)) \\
& +\eta(i, j, c)((c-i+1) \eta(i+j-c, i+k-c, h)+(h-j) \eta(i+j-c, j, h) \\
& \quad+(k-h) \eta(i+k-c, k, h)) .
\end{aligned}
$$


Proof. By part 7 of Lemma 4.2

$$
\begin{aligned}
& \sum_{a} \eta(j, k, a) \eta(i, a, c) \eta(i+a-c, j+k-a, h) \\
& =\sum_{a}(\eta(j, k, a) \eta(i, j, c)+\eta(j, k, c) \eta(c+1, k, a)) \eta(i+a-c, j+k-a, h) .
\end{aligned}
$$

Using Lemma 4.3 we obtain that

$$
\begin{aligned}
& \sum_{a} \eta(j, k, a) \eta(i, j, c) \eta(i+a-c, j+k-a, h) \\
& =\eta(i, j, c)((k-j) \eta(i+j-c, k, h)+(j-h) \eta(j, k, h) \\
& \quad+(h+c-i-k+1) \eta(i+j-c, i+k-c, h))
\end{aligned}
$$

and that

$$
\begin{aligned}
& \sum_{a} \eta(j, k, c) \eta(c+1, k, a) \eta(i+a-c, j+k-a, h) \\
& =\eta(j, k, c)((k-c-1) \eta(i+1, j+k-c-1, h)+(j-h) \eta(j, j+k-c-1, h) \\
& \quad+(h+c-i-k+1) \eta(i+1, i+k-c, h)) \\
& =\eta(j, k, c)((k-c-1) \eta(i, j+k-c, h)+(j-h) \eta(j, j+k-c, h) \\
& \quad+(h+c-i-k+1) \eta(i, i+k-c, h)) .
\end{aligned}
$$

Using these formulas and repeated application of the identity

$$
\eta(a, b, h)+\eta(b, c, h)=\eta(a, c, h)
$$

yields the result.

Theorem 4.5. For $\eta$ as defined in (2.3), the operator $g\left(e_{i} \otimes e_{j}\right)=\sum_{k} \eta(i, j, k) e_{k} \otimes$ $e_{i+j-k}$ satisifies the Yang-Baxter equation.

Proof. Let

$$
\zeta(i, j, k, c, h)=\sum_{a} \eta(j, k, a) \eta(i, a, c) \eta(i+a-c, j+k-a, h)
$$

(the left-hand side of equation (4.1)). It is easily verified that the right-hand side of equation (4.1) is then $\zeta(i+j-k, i, j, h+c-k, i+j-h)$. Now

$$
\begin{aligned}
\zeta( & +j-k, i, j, h+c-k, i+j-h) \\
= & (j+k-h-c-1) \eta(j, k, c) \eta(i-c+k, j+k-c, h) \\
& +(h-j) \eta(h, j+k-c, k) \eta(i-c+k, j+k-c, h) \\
& +(k-h) \eta(h, j+k-c, k) \eta(i-c+k, j+k-c, h) \\
& +(h+c-i-j+1) \eta(i, j, c) \eta(i+j-c, i+k-c, h) \\
& +(j-h) \eta(i+j-c, h, j) \eta(i+j-c, i+k-c, h) \\
& +(h-i) \eta(i+j-c, h, i) \eta(i+j-c, i+k-c, h) .
\end{aligned}
$$

We may then rearrange these terms one at a time using Lemma 4.2:

$$
\begin{aligned}
(j+ & k-h-c-1) \eta(j, k, c) \eta(i-c+k, j+k-c, h) \\
= & (k-c-1) \eta(j, k, c) \eta(i-c+k, j+k-c, h) \\
& \quad+(j-h)(j+k-h-c-1) \eta(j, k, c) \eta(i-c+k, j+k-c, h)
\end{aligned}
$$


and

$$
\begin{aligned}
(h-j) \eta(h, j+k-c, k) \eta(i-c+k, j+k-c, h) \\
\quad=(h-j) \eta(i+k-c, j+k-c, j) \eta(i+k-c, j+1, h) \\
\quad=(h-j) \eta(i+k-j, k, c) \eta(i+k-c, j, h) .
\end{aligned}
$$

Similarly

$$
\begin{gathered}
(k-h) \eta(h, j+k-c, k) \eta(i-c+k, j+k-c, h)=(k-h) \eta(i, j, c) \eta(i+k-c, k, h), \\
(h+c-i-j+1) \eta(i, j, c) \eta(i+j-c, i+k-c, h) \\
=(c-i+1) \eta(i, j, c) \eta(i+j-c, i+k-c, h) \\
+(h-j) \eta(i, j, c) \eta(i+j-c, i+k-c, h), \\
(j-h) \eta(i+j-c, h, j) \eta(i+j-c, i+k-c, h) \\
=(j-h) \eta(i+k-j, i, c) \eta(i+k-c, j, h)
\end{gathered}
$$

and

$$
(h-i) \eta(i+j-c, h, i) \eta(i+j-c, i+k-c, h)=(h-i) \eta(k, j, c) \eta(i+k-c, i, h) .
$$

Adding these terms and rearranging easily yields $\zeta(i, j, k, c, h)$ as required.

Finally we make some observations about invertibility and the Hecke condition. Recall that $R$ is said to be Hecke if it satisfies the condition

$$
(R-q)\left(R+q^{-1}\right)=0
$$

for some $q$.

Lemma 4.6. $\quad$ 1. $g^{2}=g$.

2. $g P=-g$.

3. $P g=g+P-I$.

Proof. The first part follows from the identity

$$
\sum_{k} \eta(i, j, k) \eta(k, i+j-k, l)=\eta(i, j, l)
$$

which is a consequence of Lemma 4.3. The second and third parts follow from the identities $\eta(j, i, k)=-\eta(i, j, k)$ and $\eta(i, j, i+j-k)=\eta(i, j, k)+\delta(k-j)-\delta(k-i)$, respectively.

Proposition 4.7. Let $\alpha$ and $\beta$ be non-zero elements of $F$. The operator $R=$ $\alpha P+\beta g$ is invertible if and only if $\alpha \neq \beta$. It is Hecke if and only if $\beta=\alpha-\alpha^{-1}$.

Proof. Using Lemma 4.6 we find that

$$
R^{2}=\beta R+\alpha(\alpha-\beta) I,
$$

and the proposition then follows immediately.

Theorem 4.8. Let $F$ be a field and let $V$ be a vector space with basis $\left\{e_{1}, \ldots, e_{n}\right\}$. Let $c \in$ End $V \otimes V$ be the linear operator

$$
c\left(e_{i} \otimes e_{j}\right)=q p^{i-j} e_{j} \otimes e_{i}+\sum_{k}\left(q-q^{-1}\right) p^{i-k} \eta(i, j, k) e_{k} \otimes e_{i+j-k} .
$$

Then $c$ is an invertible solution of the Yang-Baxter equation. 
Proof. For $p=1$ the result follows from Lemma 2.1, Theorem 3.3, Proposition 4.7 and Theorem 4.5. For more general $p$ we apply [2, Theorem 3.3].

\section{REFERENCES}

[1] E. Cremmer and J.-L. Gervais, The quantum group structure associated with non-linearly extended Virasoro algebras, Comm. Math. Phys., 134 (1990), 619-632.

[2] T. J. Hodges, On the Cremmer Gervais quantizations of $S L(n)$, Int. Math. Res. Notices, 10 (1995), 465-481.

[3] C. Kassel, Quantum Groups, Graduate Texts in Mathematics 155, Springer-Verlag New York, 1995.

Department of Mathematics, University of Cincinnati, Cincinnati, Ohio 45221-0025

E-mail address: timothy.hodges@uc.edu 